\title{
INFLUENCE OF NANOCRYSTALLINE STRUCTURE ON THE MAGNETIC PROPERTIES OF WIRES AND MICROWIRES
}

\author{
M. VÁZQUEZ ${ }^{\mathrm{a}, *}$, P. MARIN ${ }^{\mathrm{a}}$, J. ARCAS ${ }^{\mathrm{a}}$, A. HERNANDO ${ }^{\mathrm{a}}$, \\ A.P. ZHUKOV ${ }^{\mathrm{b}}$ and J. GONZÁLEZ \\ ${ }^{a}$ Instituto de Magnetismo Aplicado, UCM/RENFE, Instituto de Ciencia de \\ Materiales, CSIC, P.O. Box 155. 28230 Las Rozas, Madrid, Spain; \\ ${ }^{\mathrm{b}}$ Dept. Física Materiales, Fac. Químicas, UPV, Barrio de Alza s/n, \\ 20071 San Sebastian, Spain
}

(Received in final form 28 September 1997)

The determining factors of the outstanding magnetic properties of the so-called nanocrystalline materials are described. The discussion is based on results performed on wires and microwire materials with general composition $\mathrm{FeSiB}(\mathrm{CuNb})$ and obtained by different rapid solidification techniques. The evolution of magnetic properties (i.e., coercivity, magnetostriction) is correlated with the structural evolution associated to the corresponding heating parameters, experimentally determined by X-ray diffraction or differential scanning calorimetry. Finally, a discussion is introduced on the important role played by the structural nature of a two-phase system into the above-mentioned magnetic properties. A final analysis of some technological applications of devitrified magnetic wires and microwires is presented.

Keywords: Magnetic properties; Nanostructured materials; Amorphous and nanocrystalline alloys; Thermal treatments

\section{INTRODUCTION}

There is presently great interest in studying the magnetic properties of multiphase systems in connection with the search for materials with

\footnotetext{
* Corresponding author.
} 
outstanding magnetic properties having the final objective of using them for technological applications (Hernando and Vázquez, 1993). Here, the term multiphase must be understood from both structural and magnetic points of view. In this regard, it includes materials with amorphous, nano and microcrystalline phases, but also each one can display different magnetic nature namely ferro, antiferro, paramagnetic, etc.

In a quite general manner, such materials can be classified into two major groups: (i) Alloys of immiscible elements with magnetic nature (i.e. $\mathrm{Fe}-\mathrm{Cu}, \mathrm{Co}-\mathrm{Ag}, \mathrm{Co}-\mathrm{Cr}$, etc.) which can be obtained for example from decomposition of metastable and unstable phases of the so-called granular solids; (ii) Non-equilibrium systems. The latter can exhibit extremely hard magnetic behavior, as in the case of transition metals rare earth compounds (i.e. $\mathrm{NdFeB}$ magnets) consisting mainly of a mixture of structural phases with various magnetic characteristics (strong anisotropy supplied by the rare earth elements). But also, these systems can exhibit soft magnetic behavior as those Fe-base nanocrystals embedded in a residual amorphous phase. In both cases, sophisticated fabrication and processing techniques are required to overcome the intrinsic barrier offered by such metastable systems.

In heterogeneous systems, the magnetic behavior is mainly determined by the balanced influence of local magnetic anisotropy and exchange energy. The ferromagnetic exchange interaction tries to align parallel to each other the neighboring magnetic moments while the local structural inhomogeneities oppose to this ordering effect leading to fluctuations of the magnetic anisotropy (Hernando and Vázquez, 1993; Herzer, 1995). In this context, it can be defined as a magnetic correlation length where the magnetic moments are oriented parallel to each other by the exchange interaction. This exchange correlation length, $l_{\mathrm{ex}}$, is given by: $l_{\mathrm{ex}}=(A / K)^{1 / 2}$ where $A$ is the exchange constant and $K$ the magnetic anisotropy energy per unit volume. For those systems with strong anisotropy (i.e., rare earth compounds), a large fluctuation of local orientations of magnetization can appear, while for alloys with vanishing anisotropy a broad uniformity of the magnetization is to be expected. In the case of the Fe-base nanocrystalline systems, the optimum state (from the soft magnetic point of view) is observed when the structure consists of small nanocrystallites uniformly distributed within the whole volume being embedded in an amorphous matrix so that, the exchange interaction is large enough to overcome the 
anisotropic fluctuations of easy axes and consequently the magnetic moments easily move in unison under the application of a magnetic field.

Various Fe-base nanocrystalline systems are currently studied (i.e., FeSiBCuNb, FeZrSi, etc.) (Yoshizawa et al., 1988; Suzuki et al., 1991) mainly because of the nature of the generated crystallites which facilitates the final soft magnetic character as will be later discussed. These systems are obtained by rapid solidification techniques and most results have been reported on ribbon shaped materials. In the present work, we intend to review some previous results relevant to the devitrification process of $\mathrm{FeSiB}$ systems with small additions of elements as $\mathrm{Cu}$ and $\mathrm{Nb}$ in alloy wires obtained by in-rotating-water quenchingtechnique (Ogasawara and Ueno, 1995) (in the following, wires) and glass-coated microwires by melt-extraction (Taylor-Ulitovski technique) (Hagiwara and Inoue, 1993) (microwires in the following).

\section{ABOUT QUENCHING PROCEDURES AND MAGNETIC BEHAVIOR OF AS-CAST MATERIALS}

It is well known that heating treatments performed on amorphous materials result in the deterioration of the amorphous structure and simultaneously of their magnetically soft behavior (Köster and Herold, 1981; Fujimori, 1983). The devitrification process leads to drastic magnetic hardening, and in fact, coercivities increase several orders of magnitude upon crystallization. Nevertheless, the crystallization process is in general quite complex, involving various steps and intermediate metastable structures which give rise in some cases to very interesting magnetic behaviors. Particular compositions, as for example some $\mathrm{FeSiB}$ alloys with small addition of $\mathrm{Cu}$ and $\mathrm{Nb}$ show coercivities much smaller than in their as-cast state when they are submitted to adequate treatments resulting in partial crystallization (Yoshizawa et al., 1988; Herzer, 1989). In this section, we briefly describe the quenching techniques and subsequent treatments resulting in the devitrification process of amorphous alloy wires and microwires with composition $\mathrm{Fe}_{73.5} \mathrm{Si}_{13.5^{-}}$ $\mathrm{B}_{9} \mathrm{Cu}_{1} \mathrm{Nb}_{3}$ (in the following, $\mathrm{FeSiBCuNb}$ alloy when not specified).

Amorphous alloy wires are currently obtained by rapid quenching from the melt by plunging the molten alloy into rotating water (Vázquez and Hernando, 1996). In this way, alloy wires have typical diameters of 
around $0.1 \mathrm{~mm}$, and their compositions are commonly based in $\mathrm{Fe}$ or Co similarly to those for alloy ribbons. The composition actually determines the value of the magnetostriction constant of the wire which finally plays a decisive role in the macroscopic magnetic behavior of the samples. While highly magnetostrictive wires exhibit squared hysteresis loops with a single Barkhausen jump, those samples with vanishing magnetostriction give rise to nearly non-hysteretic loops. This is certainly a consequence of the particular distribution of the magnetoelastic anisotropy which is induced by the internal stresses quenched-in during the fabrication process coupled with the magnetostriction of the corresponding composition (Vázquez and Chen, 1995; Squire et al., 1995).

Alternatively, the other family of amorphous wires can be produced by quenching and drawing the metallic molten alloy which is coated by a glass cover. By means of this technique it is possible to fabricate microwires with diameter for the metallic nucleus ranging from about 0.5 to $20 \mu \mathrm{m}$ and glassy coating a few microns thick. The internal stresses distribution depends on the ratio thickness to diameter and hence the magnetoelastic anisotropy and the overall magnetic behavior (Vázquez and Zhukov, 1996; Velázquez et al., 1996; Chiriac et al., 1995). In Fig. 1,
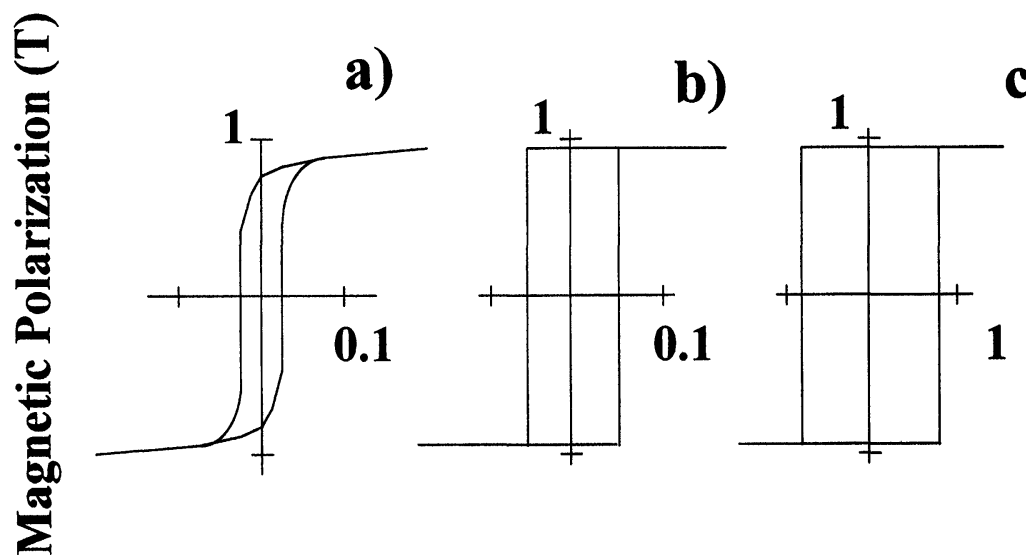

Magnetic Field (Oe)

FIGURE 1 Typical hysteresis loops of $\mathrm{FeSiB}$ magnetostrictive amorphous alloy ribbon (a), wire (b) and microwire (c). 


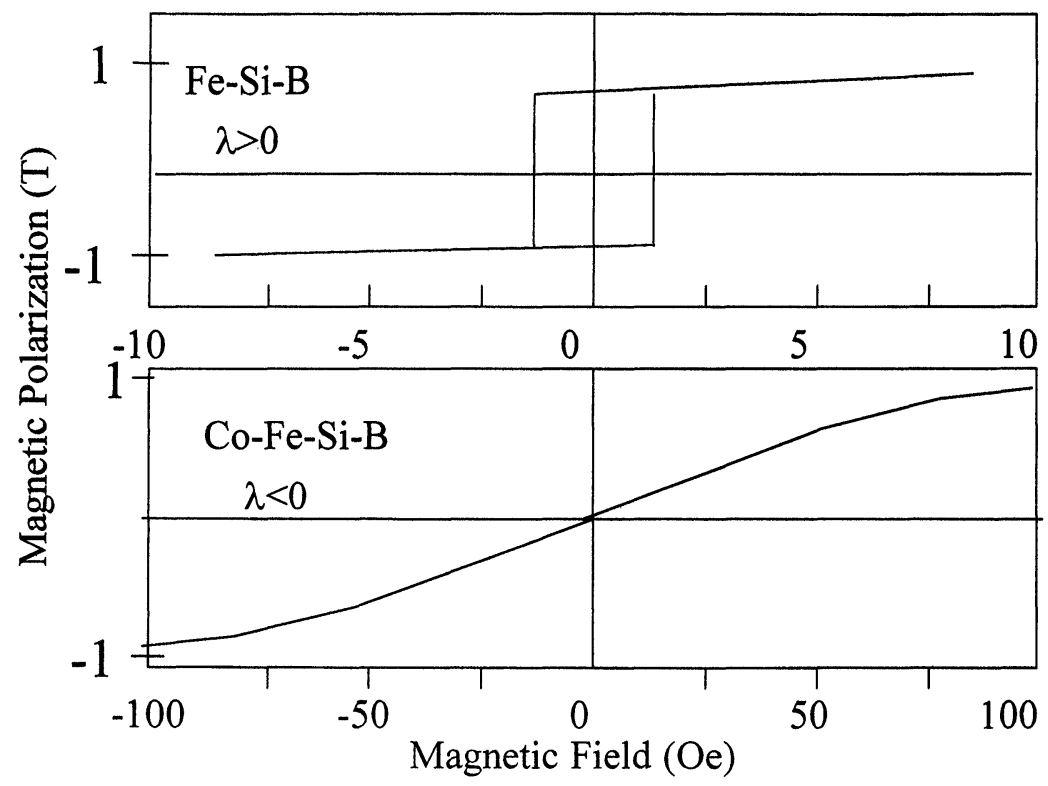

FIGURE 2 Hysteresis loops of $\mathrm{Fe}_{75} \mathrm{Si}_{15} \mathrm{~B}_{10}$ and $\mathrm{Co}_{71} \mathrm{Fe}_{4} \mathrm{Si}_{15} \mathrm{~B}_{10}$ microwires with positive and negative magnetostriction respectively.

the typical hysteresis loops for the wires and microwires obtained by the two mentioned techniques are given together with that of a conventional amorphous alloy ribbon. The differences in the hysteresis loops can be ascribed to the changes of the local stress distribution.

Figure 2 in turn shows the different behavior for $\mathrm{Fe}$ and Co base microwires with positive and negative magnetostriction respectively. As observed, there are two kinds of typical shapes for the loops and all the samples can be considered as magnetically very soft. Nevertheless, here it is clearly shown that magnetostriction (and finally the magnetoelastic anisotropy) is quite decisive in determining the loops.

\section{STRUCTURAL AND MAGNETIC EVOLUTION WITH THERMAL TREATMENTS}

The amorphous character of the structure of such materials is metastable in nature so that it can be modified by thermal treatments up to full 
devitrification. Heating is typically performed in conventional furnaces or alternatively by a current flowing along the sample that is, by current annealing. In the latter technique the temperature of the heating is not so well defined as in the first case although it has the advantage of controlling quite well annealings for very short times (Vázquez et al., 1986). The actual temperature while annealing is proportional to the current density.

Upon heating at moderate temperatures, amorphous structure relaxes at least partially resulting in the reduction of internal stresses (and the associated magnetoelastic anisotropy) and in a moderate magnetic softening (see Fig. 3(a)). Further annealing typically results in the final devitrification process and drastic magnetic hardening.

Figure 3(b) shows the evolution of the magnetic character (measured by its initial susceptibility) of a $\mathrm{FeSiBNbCu}$ wire with the current annealing (temperature) (Marin, 1995). A relative softening (i) is first observed followed by a small relative hardening (ii), a deeper softening (iii) (optimum softest behavior) and a final drastic hardening (iv). The parallel behavior with the annealing temperature in a conventional furnace is to be noted when comparing with Fig. 4.

From a structural point of view these changes in the magnetic properties can be ascribed to the following structural rearrangements: For heating temperatures of around $400-440^{\circ} \mathrm{C}$, stage (i), it corresponds to the structural relaxation of the material maintaining the amorphous character. The reduction of internal stress and magnetoelastic anisotropy brings about the loss of the rectangular shape for the hysteresis loop and accordingly reduction in coercivity (Gómez-Polo et al., 1993; Marin et al., 1994). The material loses its bistable behavior which is its most outstanding characteristics. Increasing the annealing temperature to around $460-480^{\circ} \mathrm{C}$, stage (ii), has been ascribed to the very beginning of first stage of devitrification which in this case results in the segregation of quite small $\mathrm{Cu}$ clusters inducing new but relatively small stresses (magnetoelastic anisotropy) in the amorphous regions surrounding each $\mathrm{Cu}$ cluster (Hono et al., 1993). A moderate increase of the effective magnetostriction constant has been also detected which is an additional reason for this relative hardening (Kulik et al., 1994; Vázquez et al., 1994). Stage (iii), corresponding to the state where $\alpha-\mathrm{Fe}(\mathrm{Si})$ crystallites (around $10 \mathrm{~nm}$ average grain size) are fully segregated from the initial amorphous phase and remain embedded in a residual $B$ and 

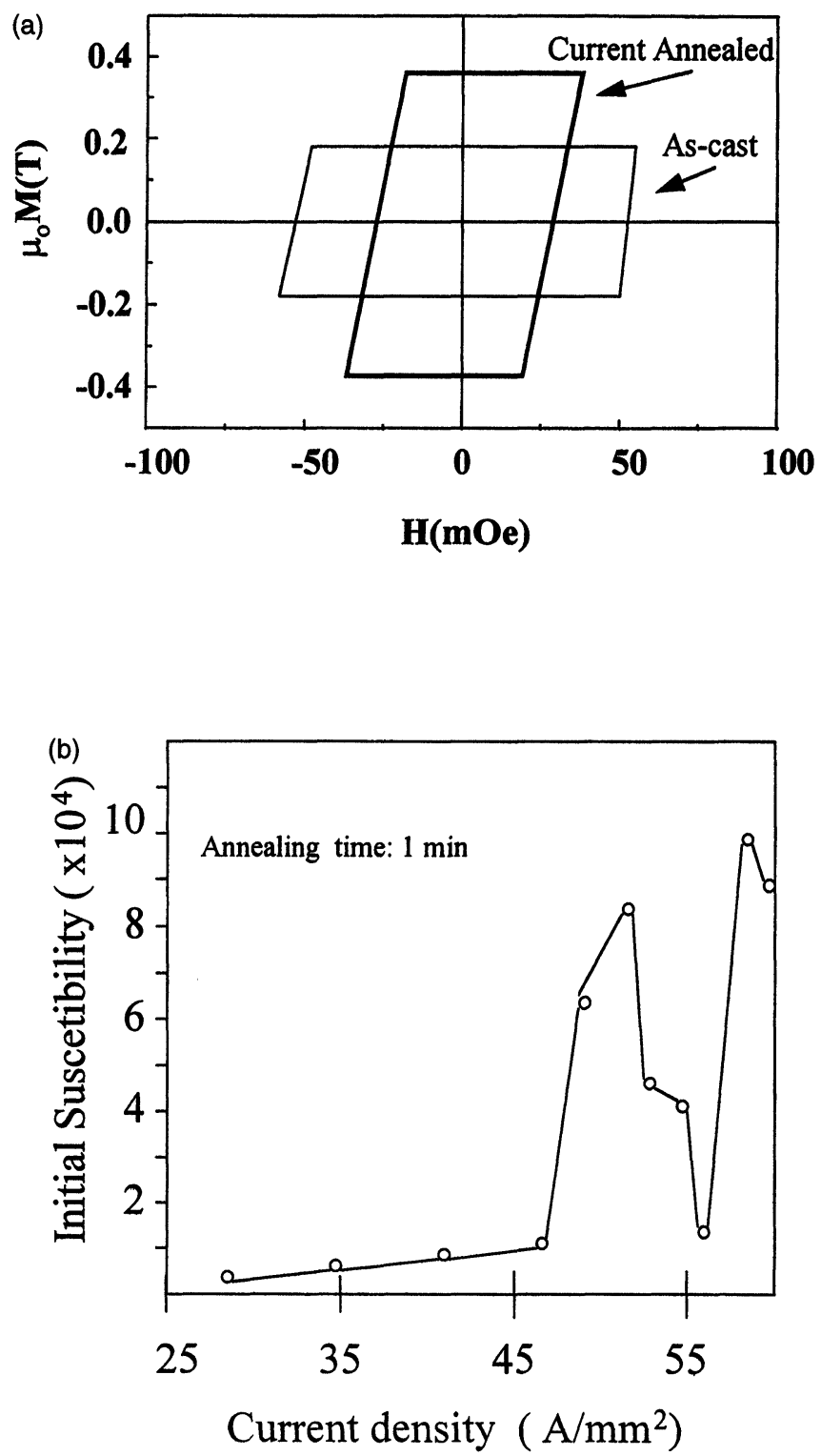

FIGURE 3 (a) The influence of current annealing $\left(32 \mathrm{~A} \mathrm{~mm}^{-2}\right.$ current density for $1 \mathrm{~h}$ ) on the hysteresis loop of a $\mathrm{CoSiB}$ wire, and (b) Evolution of the initial susceptibility for the $\mathrm{FeSiBCuNb}$ wire submitted to annealing under increasing density of current. 


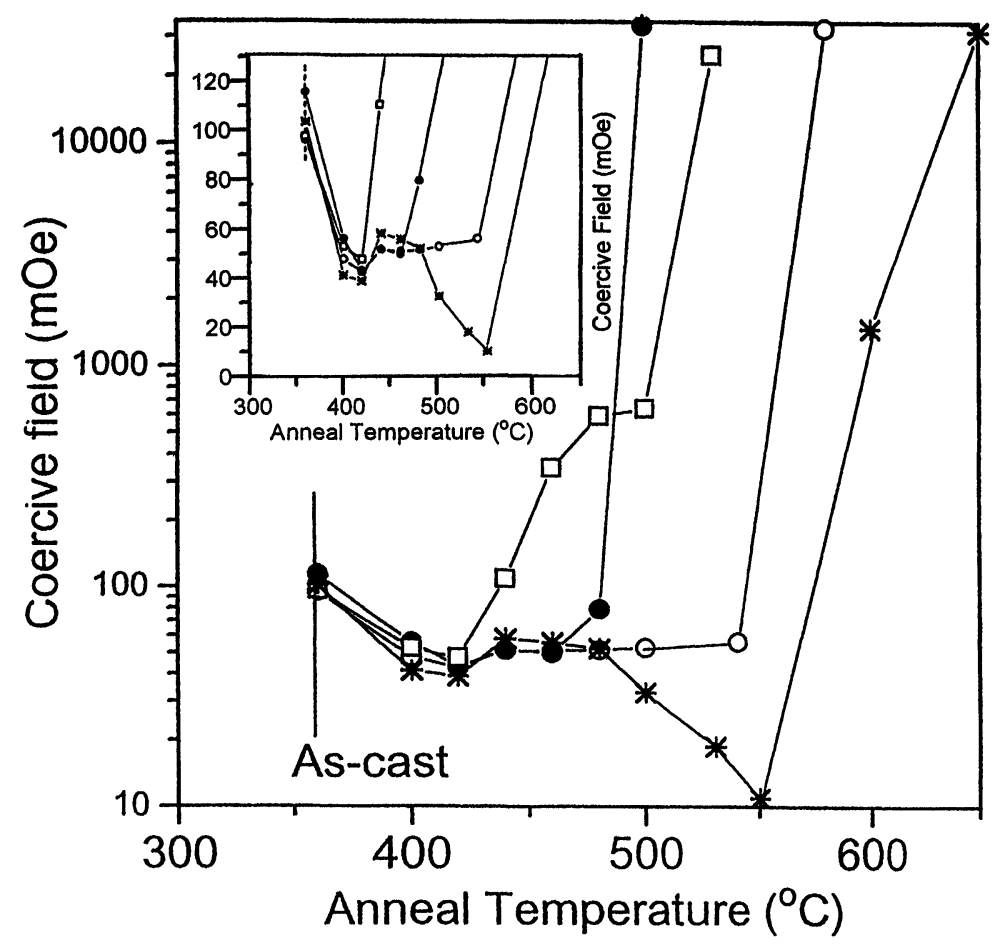

FIGURE 4 Evolution of coercivity of a $\mathrm{FeSiBNbCu}(*)$ wire in comparison with those of $\mathrm{FeSiB}(\Theta), \mathrm{FeSiBCu}(\square)$, and $\mathrm{FeSiBNb}(O)$ wires as a function of the annealing temperature.

$\mathrm{Nb}$ enriched amorphous matrix. This process takes place by increasing the number of crystallites rather than their volume (Huiseng et al., 1994). These nanocrystallites are homogeneously distributed in the whole sample and they are rather stable since such a structure remains almost unchanged for annealing treatments within the temperature range from $540^{\circ} \mathrm{C}$ to around $580^{\circ} \mathrm{C}$. This outstanding soft magnetic behavior has been interpreted to be the consequence of the averaging out of the magnetocrystalline anisotropy of the nanocrystallites, together with a compensation of the effective macroscopic magnetostriction as will be analyzed later. Here, it must be mentioned that the nanocrystalline wire can recover the bistable behavior by applying torsional stress (Gómez-Polo et al., 1993). The second devitrification process, stage (iv), starts after annealing at $600^{\circ} \mathrm{C}$ and above, when new Fe boride phases 
are segregated with rather increased size and with a final growth of the first crystallites. In this case the drastic hardening is associated to the heterogeneous size of the different grains as well as to the different nature of such grains so exhibiting various magnetic anisotropy strengths which finally results in the strong pinning centers for the domain walls.

In order to investigate the influence of the various elements in the alloy, different alloy wires with different compositions have been studied. They are $\mathrm{Fe}_{75} \mathrm{Si}_{15} \mathrm{~B}_{10}, \mathrm{Fe}_{75} \mathrm{Si}_{14} \mathrm{~B}_{10} \mathrm{Cu}_{1}$ and $\mathrm{Fe}_{73} \mathrm{Si}_{14} \mathrm{~B}_{11} \mathrm{Nb}_{3}$ apart from $\mathrm{FeSiBCuNb}$ which has been described before. The evolution of coercivity with the annealing temperature is shown in Fig. 4.

Figure 5 shows the evolution of the nature and grain size of the various crystallites for the series of alloy wires shown in Fig. 4 as deduced from $\mathrm{X}$-ray diffractometry. The case of the devitrification of the FeSiB wire is a typical one in the sense that after the relaxation of the amorphous structure (reduction of coercivity) there is a drastic increase of coercivity

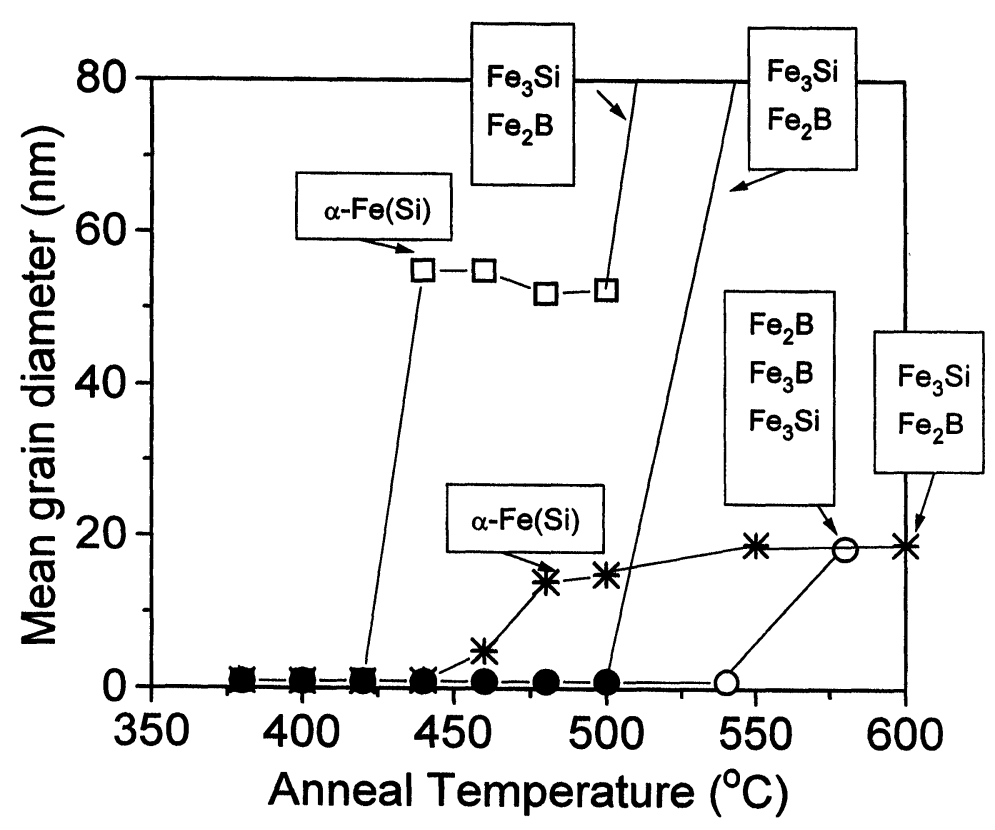

FIGURE 5 Evolution of nature and grain size of crystallites for wires with different composition: $\mathrm{FeSiB}(\bigcirc), \mathrm{FeSiBCu}(\square), \mathrm{FeSiBNb}(\bigcirc)$ and $\mathrm{FeSiBCuNb}(*)$. 
resulting from the $\mathrm{Fe}$ crystallites with grain size large enough. In the $\mathrm{FeSiBCu}$ alloy, the presence of an intermediate devitrification stage can be observed between the two main crystallization processes mentioned above. Finally, the presence of $\mathrm{Nb}$ delays the second crystallization process so that, in the case of the FeSiBCuNb alloy both effects appear together namely, the existence of two crystallization processes (caused by $\mathrm{Cu}$ ), and the well-defined separation between them (originated by $\mathrm{Nb}$ ). In this latter case, the existence of homogeneous and stable $\mathrm{Fe}$ nanocrystallites is well defined. Figure 6 shows additional results concerning the influence of the elements on the differential scanning calorimetry spectra supporting the role of each element which is analyzed in detail elsewhere (Marin et al., submitted).
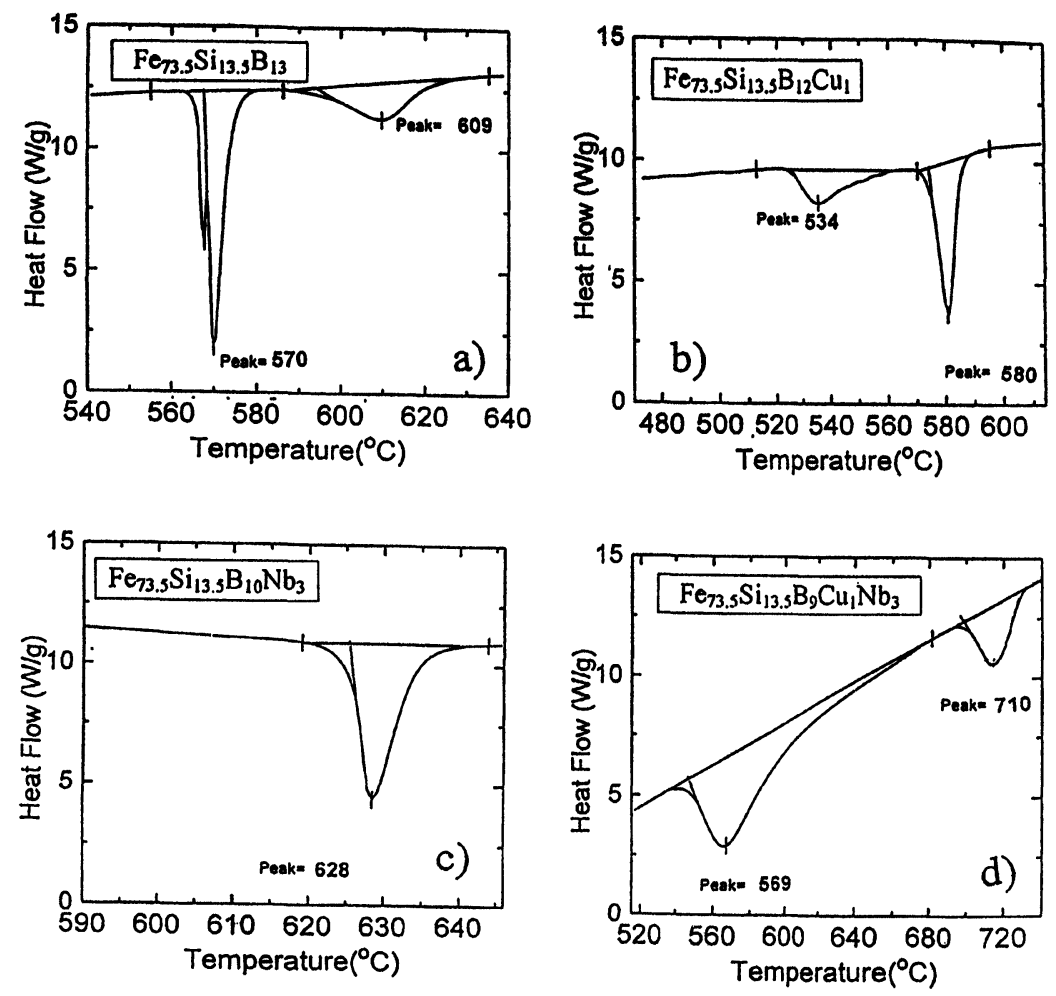

FIGURE 6 DSC spectra of indicated alloy wires. 


\section{MAGNETIC BEHAVIOR OF DEVITRIFIED MICROWIRES}

A quite interesting magnetic behavior is observed for the $\mathrm{FeSiBCuNb}$ microwire (same composition as that of the conventional amorphous wire) covered by glass coating. Thermal treatments performed in these samples lead, as in the previous case, to similar devitrification processes but the magnetization process differs from that of those wires. As can be observed in Fig. 7, the coercivity can be strongly reduced by annealing at temperatures ranging around $560^{\circ} \mathrm{C}$ but when annealing at the highest temperatures (up to about $775^{\circ} \mathrm{C}$ ) the typical hardening appears. It can be observed that in the optimum soft magnetic state, coercivity is reduced down to around 0.1 Oe while after the final heating coercivity reaches values around 60-80 Oe (Arcas et al., 1996). Few things could be
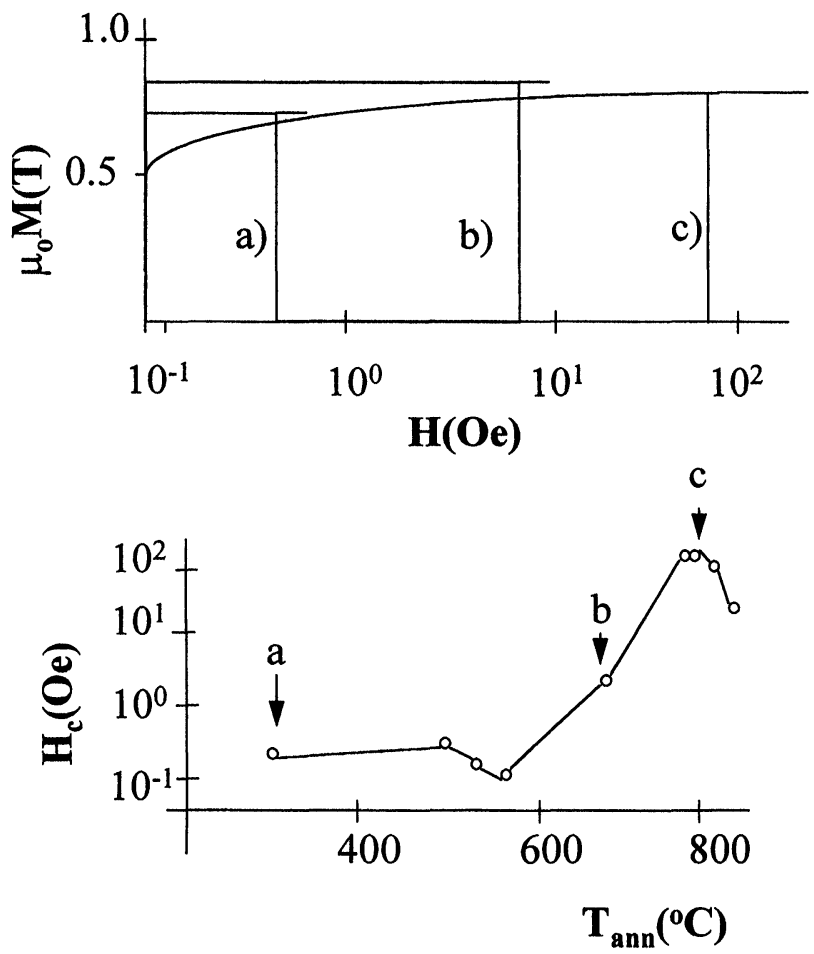

FIGURE 7 Magnetization curves and coercivities of FeSiBCuNb microwires submitted to heatings at indicated temperatures (note the semi-logarithmic scale for the coercivity). Bistability remains up to annealing at $750^{\circ} \mathrm{C}$. 
added in comparison with the previously analyzed wires regarding the structural changes while the different devitrification processes are achieved. Only, the effect of heatings is somehow shielded by the glass coating so that, the annealing temperatures for which "nanocrystalline" state as well as the second crystallization process are completed are slightly higher than in the previous case.

Nevertheless, from the magnetic point of view it is very important to emphasize that it is possible to maintain the square shape of the loops even at the optimum soft state as well as after annealing up to around $750^{\circ} \mathrm{C}$ (see Fig. 7) with an increase of almost 3 orders of magnitude for the coercivity. The maintenance of such outstanding behavior must be ascribed to the presence of the quite strong mechanical stresses at the as-cast state which cannot be completely relieved during the first treatments. Also, it might be that upon nano and further crystallization some texture will be present preserving an effective magnetic anisotropy with axial easy axis so that, magnetization reversal takes place in a single step and maintaining the square loop like in as-cast material.

\section{ABOUT THE MAGNETIC HARDENING-SOFTENING MECHANISMS DURING THE DEVITRIFICATION PROCESS}

In Sections 3 and 4 we have presented the main structural and magnetic stages during the devitrification processes of amorphous wires and microwires. It is possible to observe that for each change of structural character a different magnetic behavior is found. This is finally, as we are going to discuss, a consequence of the competition between structural anisotropy and exchange interaction. Fully quantitative derivations of that competition are nevertheless very hard to attain, but at least a qualitative description is being presented. For such a description it is interesting to consider the exchange correlation length, $l_{\mathrm{ex}}=(A / K)^{1 / 2}$ previously introduced as a balance parameter between exchange interaction and locally fluctuating structural anisotropy.

First of all, it is possible to define a structural correlation length, $l_{\mathrm{st}}$, in a single-phase system, as the grain size of the crystallites (about $10 \AA$ for amorphous systems, $10 \mathrm{~nm}$ for nanocrystals, $1 \mu \mathrm{m}$ for microcrystals and $1 \mathrm{~mm}$ or larger for polycrystals in general). Within this structural 
correlation length, magnetic moments follow the orientation determined by the crystalline anisotropy inside each crystallite. Typically, at the border between grains there is an additional contribution to local anisotropy from magnetoelastic origin (stresses from crystal imperfections such as dislocations, etc.) besides dipolar local interactions. All these contributions determine a given fluctuation of local magneto-structural anisotropy.

The case of amorphous structure can be treated as a single-phase system where this structural correlation length is extremely small (a few atomic distances) so that, the effective anisotropy arises from magnetoelastic origin and in fact, the exchange correlation length is determined not by the crystalline structure but by the magnetoelastic structure (size of regions with different character for the mechanical stresses). For example, the correlation length for the amorphous structure in as-cast magnetostrictive $\mathrm{Fe}$-base alloy wires corresponding to the intrinsical magnetoelastic anisotropy can be taken as $l_{\mathrm{ex}}=30 \mathrm{~nm}$ with $A=4 \times 10^{-12} \mathrm{~J} \mathrm{~m}^{-1}, \lambda=3 \times 10^{-5}$ and the average strength of internal stresses as $80 \mathrm{MPa}$. After relaxation of the amorphous structure by heating, but maintaining its amorphous nature, those stresses relax and the exchange correlation length increases to about $60 \mathrm{~nm}$. In the case of Co-base alloys with vanishing magnetostriction, these correlation lengths can take values up to around 500 and $1.000 \mathrm{~nm}$ in their as-cast and relaxed states, respectively (note that these values roughly correspond to the width of domain walls).

According to the classical theory considering planar walls, the coercivity can be expressed as:

$$
H_{\mathrm{c}}=\left(\Delta K / 2 \mu_{0} M_{\mathrm{s}}\right) l_{\mathrm{ex}} / l_{\mathrm{st}},
$$

where $l_{\text {st }}$ is associated to a structural correlation length which denotes an average distance between pinning centers $\left(l_{\text {ex }} \ll l_{\text {st }}\right)$ and $\Delta K$ actually represents the amplitude of the fluctuating anisotropy within the structural correlation length $l_{\text {st }}$.

The mentioned structural relaxation within amorphous structure leads to a reduction of the coercivity through the reduction of $\Delta K$ but also to an increase of $l_{\mathrm{ex}}$ so that, finally coercivity increases roughly with the square root of $K$ as amorphous structural relaxation proceeds. This magnetic softening is observed in conventional amorphous material 
(ribbons) but it is enhanced in the case of the wire material due to the additional effect of losing the bistable character by the decrease of the magnetoelastic anisotropy. In the experimental results presented in Fig. 4, it corresponds to those after the annealings at temperatures between $400^{\circ} \mathrm{C}$ and $440^{\circ} \mathrm{C}$.

What is more astonishing is the relative hardening after annealing at around $460-480^{\circ} \mathrm{C}$, where no real crystallization by X-ray diffraction is detected but some atomic rearrangements ascribed to the segregation of quite small $\mathrm{Cu}$ clusters. As deduced from the experimental results, there is a clear increase of the anisotropy field when magnetizing axially indicating an increase of a somehow induced magnetic anisotropy. This anisotropy must be ascribed to the magnetoelastic anisotropy induced by local stresses in the amorphous matrix arising while $\mathrm{Cu}$ clusters segregate. Furthermore, in the very initial stages of crystallization of $\alpha-\mathrm{Fe}(\mathrm{Si})$ nanocrystallites, they are occupying very small volume fraction so that they are not physically touching but magnetically coupled through the ferromagnetic amorphous matrix. These two effects actually harden the material. Coercivity for example, in Eq. (1), increases owing to the increase of the magnetoelastic anisotropy but also due to the decrease of the structural correlation length, $l_{\text {st }}$ which now can be ascribed to the average distance between $\mathrm{Cu}$ or $\alpha-\mathrm{Fe}(\mathrm{Si})$ clusters.

This particular state can be considered as either that of a single phase material with a certain amount of inclusions (magnetic and non-magnetic) or that of a multiphase material where the volume fraction of one of the phases is much greater than that of the rest.

In a two-phase system, there is an interaction between magnetic moments from the two systems. In the case when the exchange constant is similar for both systems, we propose the following relationship between exchange correlation lengths and anisotropy constants:

$$
l_{\text {ex }, \mathrm{am}}=l_{\mathrm{ex}, \mathrm{nc}}\left(K_{\mathrm{nc}} / K_{\mathrm{am}}\right)^{1 / 2},
$$

where $l_{\text {ex,am }}$ is the length within the amorphous matrix to which the ordering effect of the magnetocrystalline anisotropy, $K_{\mathrm{nc}}$, of a single nanocrystallite extents. Similarly, $l_{\text {ex,nc }}$ is the length inside the crystallite to which the effect of the magnetoelastic order from the amorphous matrix extents.

In the previous case with the existence of the amorphous matrix and the inclusions of magnetic clusters, the grain size of crystallites $l_{\mathrm{st}, \mathrm{nc}}$ is 
quite small compared with that of $l_{\text {ex,nc }}$, so that the crystallites can be taken as isolated pinning centers. $l_{\text {st,am }}$ corresponds to the distance between crystallites which is much larger than $l_{\text {ex,am. }}$. A transition appears when crystallites grow in size and number so that $l_{\mathrm{st}, \mathrm{am}} \approx l_{\mathrm{ex}, \mathrm{am}}$ which means that crystallites see each other magnetically.

As the heating is performed at higher temperatures the new $\alpha-\mathrm{Fe}(\mathrm{Si})$ phase increases its volume fraction. The case of the optimum nanocrystalline state, corresponding to heating at around $540^{\circ} \mathrm{C}$, is probably that to which most attention has been paid in the last years also because of the potential technological application of such soft materials. It has been mainly analyzed by Herzer who considers essentially this softness as associated to the negligible magnetic anisotropy. As experimentally shown, in this case the material consists largely of $\alpha-\mathrm{Fe}(\mathrm{Si})$ nanocrystallites (about $10 \mathrm{~nm}$ average grain size) occupying around $75 \%$ of the total volume. The rest mainly consists of a residual amorphous matrix reduced in $\mathrm{Fe}$ and enriched in $\mathrm{B}$ and $\mathrm{Nb}$ in comparison with the initial material. This matrix actually is surrounding the nanocrystallites and can be mostly considered as a tiny surface covering those crystallites. Consequently, the material consists of a two-phase system with nanocrystals and the matrix, and the effective magnetic anisotropy contains magnetocrystalline and magnetoelastic terms.

According to the random anisotropy model (Alben et al., 1978; Herzer, 1989) the magnetocrystalline anisotropy can be taken as

$$
K_{\mathrm{mc}}=K N^{-1 / 2},
$$

the smallness of which arises from the averaging effect due to the random distribution of the orientation of easy axes for the magnetocrystalline anisotropy, $K$, of the crystallites. $N$ denotes the number of crystallites inside the volume reached by the exchange correlation length.

The effective magnetoelastic anisotropy is expressed as

$$
K_{\mathrm{me}}=(3 / 2)\left(\lambda_{\mathrm{am}} V_{\mathrm{am}}+\lambda_{\mathrm{nc}} V_{\mathrm{nc}}\right) \sigma,
$$

where the effective magnetostriction contains contributions from the crystallites ( $\lambda_{\mathrm{nc}}$ with negative sign) and from the amorphous matrix $\left(\lambda_{\mathrm{am}}\right.$ with positive sign) which result in a compensation effect. The evolution of magnetostriction with annealing is shown in Fig. 8 where such compensation effect is observed for the $\mathrm{FeSiBCuNb}$ wire. Also in this figure, 
a quite particular behavior of the magnetostriction is shown demonstrating the existence of phases with different sign for the magnetostriction (Hernando et al., 1997). Figure 8(b) shows the evolution of the magnetostrictive deformation with axial applied field for different values of transverse field simultaneously applied for the $\mathrm{Fe}_{73.5^{-}}$ $\mathrm{Si}_{11} \mathrm{~B}_{15.5} \mathrm{Nb}_{3} \mathrm{Cu}_{1}$ alloy. If the material consists of a two-phase system, each with different magnetostriction (even in sign) the magnetostrictive deformation can be expressed as

$$
\Delta \varepsilon=3 / 2\left(\lambda_{\mathrm{am}} V_{\mathrm{am}} \alpha+\lambda_{\mathrm{nc}} V_{\mathrm{nc}} \beta\right),
$$

where $V_{\text {am }}$ and $V_{\text {nc }}$ are the volume fractions with positive (residual amorphous matrix) and negative magnetostriction (nanocrystalline phase), and $\alpha$ and $\beta$ the volume fractions in each one of those different magnetostrictive regions where the easy axis is parallel to the axis of the material. The contributions of the volume fractions $\alpha V_{\mathrm{am}}$ and $(1-\beta) V_{\mathrm{nc}}$ to the magnetostrictive deformation is zero since only magnetization rotations make a real contribution. From Eq. (5) it can be deduced that when $\beta \gg \alpha$ the total deformation under application of axial field can be

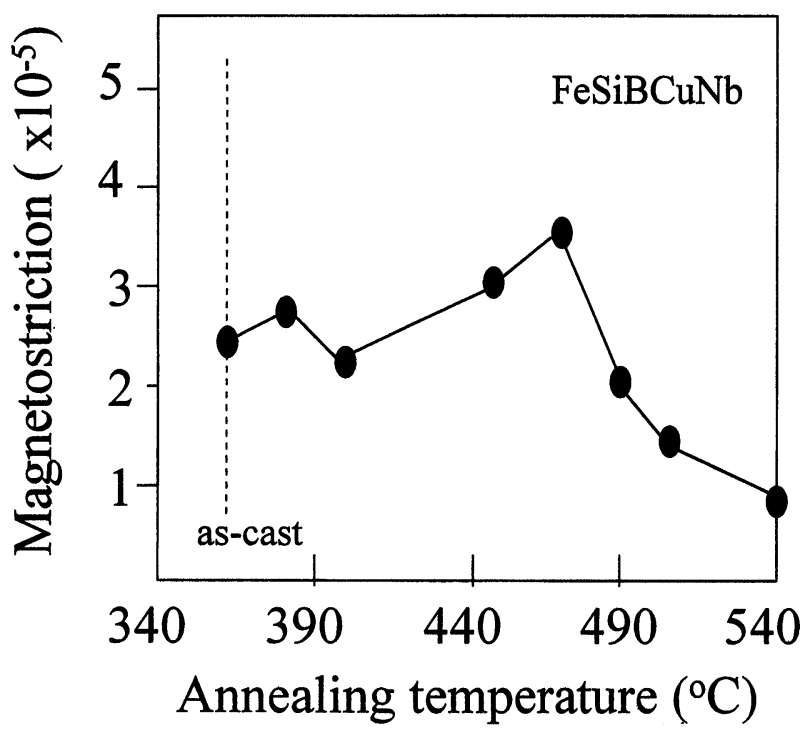

FIGURE 8(a) 


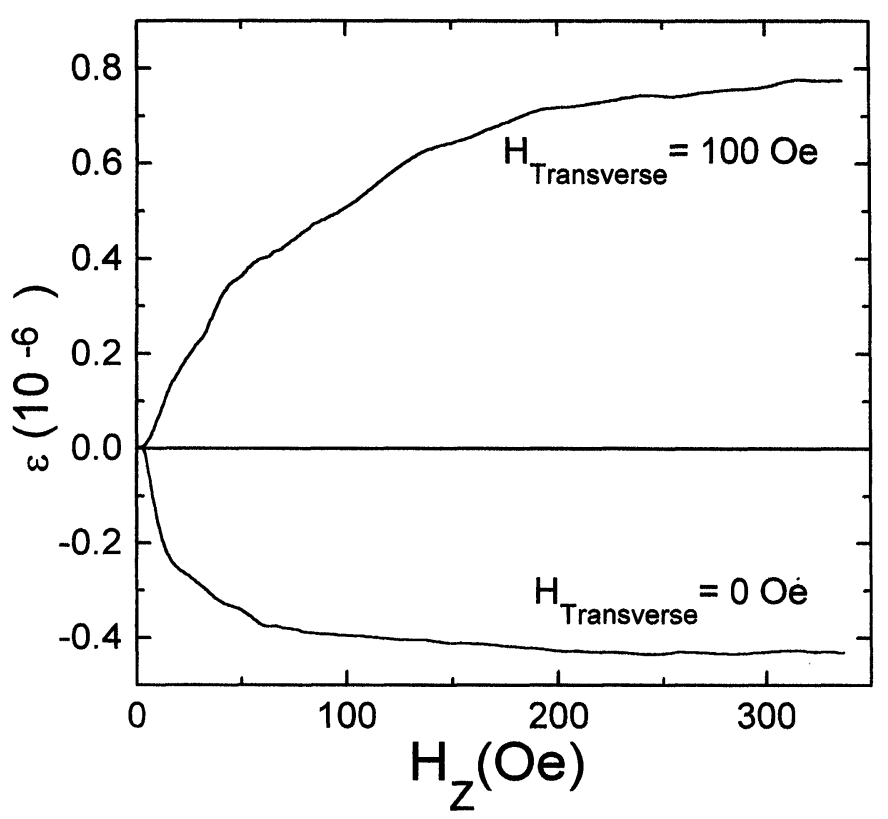

FIGURE 8(b)

FIGURE 8 (a) Change of magnetostriction with increasing annealing temperature, and (b) evolution of the magnetostrictive deformation with axial field in the presence of saturating and zero transverse field for a two-phase alloy with positive saturation magnetostriction (see text).

negative. But when the experiment is performed under a saturating DC transverse field, $\alpha=0$ and $\beta=1$ so that the final deformation is positive. This is the experimental case shown in the figure for the $\mathrm{Fe}_{73.5} \mathrm{Si}_{11^{-}}$ $\mathrm{B}_{15.5} \mathrm{Nb}_{3} \mathrm{Cu}_{1}$ alloy. The important conclusion is that this result experimentally demonstrates the existence of phases with different sign of magnetostriction (Arcas et al., 1998; Hernando et al., 1995).

The second crystallization occurs after annealing at temperatures higher than about $600^{\circ} \mathrm{C}$. The outstanding soft magnetic behavior is lost dramatically (i.e. increase of the coercivity by around three orders of magnitude) as a consequence of two effects: the appearance of new boride phases (intrinsically harder, with exchange correlation length not larger than about $5 \mathrm{~nm}$ ) and the final growth of the nanocrystallites. Both result in a heterogeneity effect of the structure, and according to Eq. (1) to an increase of the total magnetic anisotropy (magnetocrystalline and 
magnetoelastic) as well as to a decrease of the effective exchange correlation length. The strong fluctuating structural correlation length is the origin for that hardening.

\section{TWO EXAMPLES OF TECHNOLOGICAL APPLICATIONS USING NANOCRYSTALLINE WIRES AND MICROWIRES}

\subsection{Magnetic Field Sensing and Giant Magnetoimpedance in Nanocrystalline Wires and Microwires}

The magnetoimpedance effect has been recently discovered in soft amorphous wires with very high circular permeability (Beach and Berkowitz, 1994; Panina and Mohri, 1995; Velázquez et al., 1995). It consists of the large relative change (up to around $400 \%$ ) of the impedance (both real and imaginary parts) upon application of DC magnetic field or stress. In order to measure the impedance, typically a low density current flows along the sample having a frequency in the range of $\mathrm{MHz}$. This effect has been detected in nearly zero magnetostriction Co-base samples in the shape of wire, ribbon and thin film. It has been interpreted as arising from the classical skin effect the penetration depth of which defined as

$$
\delta=(\rho / \pi \mu f)^{1 / 2}
$$

$\rho$ being the resistivity, $f$ the frequency and $\mu$ the transverse permeability. Upon application of a DC field, the permeability decreases, and accordingly $\delta$ increases until it reaches the radius of the wire which finally results in an effective change of the impedance.

Here, we wanted just to describe shortly the influence of the nanocrystallization on the magnetoimpedance effect. This has been recently reported for $\mathrm{FeSiBCuNb}$ wires after being submitted to the heatings mentioned above (Knobel et al., 1995; 1996). In the optimum soft magnetic nanocrystalline state (annealing at $540^{\circ} \mathrm{C}$ ), a maximum magnetoimpedance ratio of less than $100 \%$ is reached what is actually smaller than for the mentioned Co-base material. Nevertheless, it is interesting to mention that after starting the second crystallization process (annealing at $600^{\circ} \mathrm{C}$ ), this ratio is observed to increase up to 
around $200 \%$. Results after annealing at higher temperature indicate a much more moderate effect. This large magnetoimpedance effect is ascribed to the fact that permeability is still relatively large, while resistivity decreases notably giving a balance effect which results in an improved magnetoimpedance ratio as shown in Fig. 9.

The importance of this effect is related with its potential application in magnetic field sensors. In fact, several prototype devices have been developed using this phenomenon as position, field, or current sensors (Vázquez et al., 1997). In the case of nanocrystalline wire materials, it can be employed especially in some particular devices where the response at relatively elevated temperatures is required to be constant. In this case, the nanocrystalline structure is not altered in comparison with that of the initial amorphous state.

\subsection{Magnetic Tags Using Amorphous and Nanocrystalline Microwires}

An application which has been successfully developed consists in the use of $\mathrm{FeSiBCuNb}$ microwires submitted to different heating treatments for

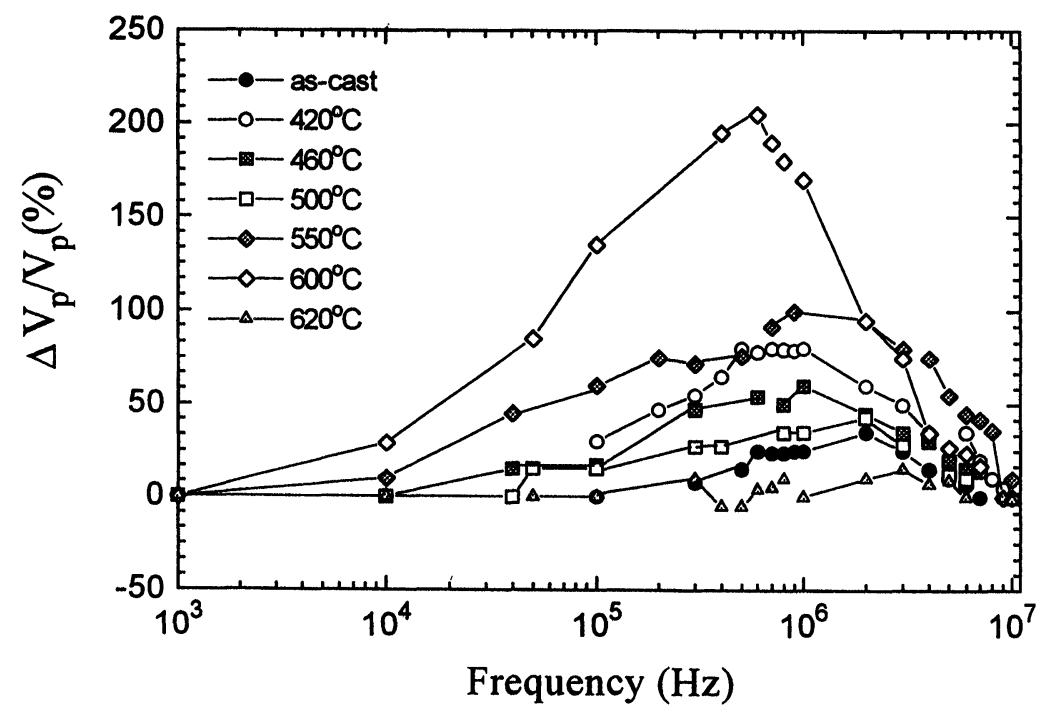

FIGURE 9 Frequency dependence of the relative change in voltage for the FeSiB$\mathrm{CuNb}$ wire (magneto-impedance effect for a fixed value of the current). The maximum is observed after heating at $600^{\circ} \mathrm{C}$. 
sensing elements in magnetic tags with a certain storage capacity. The tag contains several microwires with well-differentiated coercivities, all of them characterized by a square or bistable magnetic loop. Once the magnetic tag is submitted to an increasing magnetic field, each particular microwire reverses magnetization resulting in an electrical signal on a coil system (Vázquez et al., 1996). This is schematically shown in Fig. 10 for a set of three microwires.

The importance of using the above-described microwires lies in the fact that as shown, it is possible to tailor the material within a wide range of coercivities (from around 0.1 to almost $100 \mathrm{Oe}$ ) just by heating at adequate temperatures maintaining the square shape of the hysteresis loop (see Fig. 7).
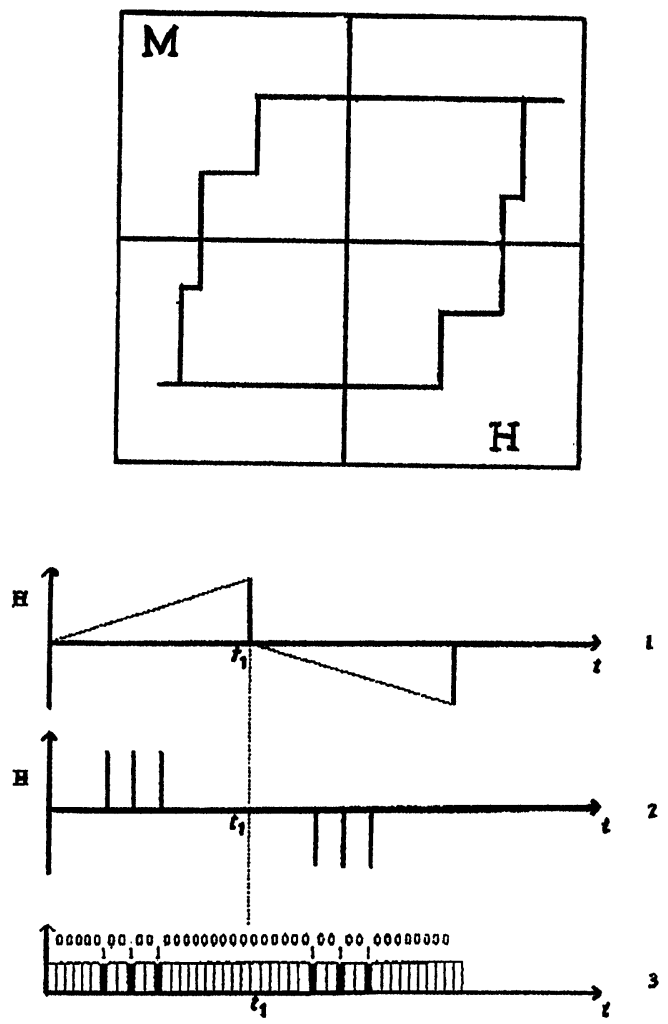

FIGURE 10 Schematic representation of the working system of a magnetic tag as described in the text. 


\section{CONCLUSION}

In this work the influence of the heating treatments on the structural and magnetic behavior of wires and microwires obtained by two rapid solidification techniques having typical composition $\mathrm{FeSiBCuNb}$ has been reviewed. Both kinds of characteristics are strongly correlated. In fact, when the processing techniques are sophisticated enough to control the structure, it is accordingly possible to tailor the magnetic properties. The magnetic softening-hardening mechanisms have been proved to strongly correlate with the parallel structural changes.

One important difference between the described wires and microwires is that the latter maintains the square loop after nanocrystallization and even well beyond the second crystallization process which is ascribed to the much stronger intrinsical stresses of the microwires. Finally, two particular applications making use of nanocrystalline wires and microwires are introduced.

\section{Acknowledgments}

The authors are deeply indebted to Prof. H.A. Davies and to Dr. V. Larin for their guidance in the preparation of amorphous wires and microwires respectively. They also wish to thank the researchers at the IMA not included as coauthors but making important contributions to this report. The work has been supported by the Spanish CICYT under project MAT95/0273.

\section{References}

Alben, R., Becker, J.J. and Chi, M.C. (1978). Random anisotropy in amorphous ferromagnets. Journal of Applied Physics, 49(3), 1653-1658.

Arcas, J., Gomez-Polo, C., Zhukov, A.P., Vázquez, M., Larin, V. and Hernando, A. (1996). Structural, mechanical and magnetic properties in heat-treated $\mathrm{Fe}_{73.5} \mathrm{Si}_{22.5-x^{-}}$ $\mathrm{B}_{x} \mathrm{Nb}_{3} \mathrm{Cu}_{1}(6 \leq x \leq 12)$ alloy ribbons. Nanostructured Materials, 7, 823-834.

Arcas, J., Hernando, A., Garcia-Beneytez, J.M. and Vázquez, M. (1998). Spatial fluctuations of magnetostriction in a nanostructured systems. Journal of Magnetism and Magnetic Materials, 186, 283-287.

Beach, R.S. and Berkowitz, A. (1994). Giant magnetic field dependence impedance of amorphous FeCoSiB wire. Applied Physic Letters, 64, 3652-3654.

Chiriac, H., Ovari, T.A. and Pop, G. (1995). Internal stress distribution in glass-covered amorphous magnetic wires. Physical Review B, 52, $10104-10113$.

Fujimori, H. (1983). Magnetic anisotropy. In Amorphous Metallic Alloys, edited by, F.E. Luborsky, pp. 300-316. London, Butterworths. 
Gómez-Polo, C., Olofinjana, A.O., Marin, P., Vázquez, M. and Davies, H.A. (1993). The influence of nanocrystalline microstructure on the magnetic properties of a wire shaped ferromagnetic alloy. IEEE Transactions on Magnetics, 29, 2673-2675.

Hagiwara, M. and Inoue, A. (1993). Production technique of alloy wires by rapid solidification. In Rapidly Solidified Alloys, ed. H.H. Liebermann, pp. 139-156. New York, Marcel Dekker.

Hernando, A. and Vázquez, M. (1993). Engineering properties of rapidly solidified alloys. In Rapidly Solidified Alloys, ed. H.H. Liebermann, pp. 553-590. New York, Marcel Dekker.

Hernando, A., Vázquez, M., Kulik, T. and Prados, C. (1995). Analysis of the dependence of spin-spin correlations on the thermal treatment of nanocrystalline materials. Physical Review B, 51, 3581-3586.

Hernando, A., Vázquez, M., Arcas, J. and García-Beneytez, J.M. (1997). Magnetostriction in heterogeneous nanocrystalline materials. Philosophical Magazine B, 76, 441-446.

Herzer, G. (1995). Soft magnetic nanocrystalline materials. Scripta Metallurgica, 33, 1741-1756.

Herzer, G. (1989). Grain structure and magnetism of nanocrystalline ferromagnets. IEEE Transactions on Magnetics, 25, 3327-3329.

Hono, K., Li, J.L., Ueki, Y., Inoue, A. and Sakurai, T. (1993). Atom probe study of the crystallization process of an $\mathrm{FeSiBNbCu}$ amorphous alloy. Applied Surface Science, 67, 398-406.

Huiseng, Y., Guochao, T., Xiaotao, X., Zuxiong, X. and Ruzhang, M. (1994). Structure of the amorphous phase during crystallization of $\mathrm{Fe}_{73.5} \mathrm{Cu}_{1} \mathrm{Nb}_{3} \mathrm{Si}_{13.5} \mathrm{~B}_{9}$ alloy. Journal of Magnetism and Magnetic Materials, 138, 94-98.

Knobel, M., Sánchez, M.L., Marín, P., Gómez-Polo, C., Vázquez, M. and Hernando, A. (1995). Influence of nanocrystallization on the magneto-impedance effect in FeCuNbSiB amorphous wires. IEEE Transactions on Magnetics, 31, 4009-4011.

Knobel, M., Sánchez, M.L., Gómez-Polo, C., Marín, P., Vázquez, M. and Hernando, A. (1996). Giant magneto-impedance effect in nanostructured magnetic wires. Journal of Applied Physics, 79, 1646-1654.

Köster, U. and Herold, U. (1981). Crystallization of metallic glasses. In Glassy Metals I edited by H. Güntherodt and H. Beck, pp. 225-260. Berlin, Springer Verlag.

Kulik, T., Hernando, A. and Vázquez, M. (1994). Correlation between structure and the magnetic properties of amorphous and nanocrystalline $\mathrm{Fe}_{73.5} \mathrm{Cu}_{1} \mathrm{Nb}_{3} \mathrm{Si}_{22.5-x} \mathrm{~B}_{x}$ alloys. Journal of Magnetism and Magnetic Materials, 133, 310-313.

Marin, P., Olofinjana, A.O., Vázquez, M. and Davies, H.A. (1994). Influence of the as-cast state on the crystallization process and magnetic properties for $\mathrm{FeSiBCuNb}$ wires. IEEE Transactions on Magnetics, 30, 4794-4797.

Marín, P. (1995). Doctoral Thesis. Evolución de las propiedades magnéticas durante el proceso de nanocristalización de hilos amorfos de composición $\mathrm{FeSiBCuNb}$. University Complutense, Madrid.

Marin, P., Vázquez, M., Olofinjana, A.O. and Davies, H.A. (submitted). The influence of $\mathrm{Cu}$ and $\mathrm{Nb}$ on relaxation and nanocrystallization process of amorphous $\mathrm{FeSiB}$ $(\mathrm{CuNb})$ amorphous wires.

Ogasawara, I. and Ueno, S. (1995). Preparation and properties of amorphous wires. IEEE Transactions on Magnetics, 31, 1219-1223.

Panina, L.V. and Mohri, K. (1994). Magneto-impedance effect in amorphous wires. Applied Physics Letters, 65, 1189-1191.

Squire, P., Atkinson, D. and Atalay, S. (1995). Magnetostrictive and magnetoelastic properties of rapidly quenched wire. IEEE Transactions on Magnetics, 31, 1239-1248.

Suzuki, K., Makino, A., Kataoka, N., Inoue, A. and Masumoto, T. (1991). High saturation magnetization and soft magnetic properties of bcc FeZrB alloys with ultrafine grain structure. Materials Transactions, JIM-31, 743-746. 
Vázquez, M., González, J. and Hernando, A. (1986). Induced magnetic anisotropy and change of the magnetostriction by current annealing in Co based amorphous. Journal of Magnetism and Magnetic Materials, 53, 323-329.

Vázquez, M., Marin, P., Davies, H.A. and Olofinjana, A.O. (1994). Magnetic hardening of $\mathrm{FeSiBCuNb}$ ribbons and wires during the first stage of crystallization to a nanophase structure. Applied Physics Letters, 64, 3184-3186.

Vázquez, M. and Chen, D.-X. (1995). The magnetization reversal process in amorphous wires. IEEE Transactions on Magnetics, 31, 1229-1238.

Vázquez, M. and Hernando, A. (1996). A soft magnetic wires for sensor applications. Journal of Physics D: Applied Physics, 29, 939-949.

Vázquez, M., Zhukov, A.P., Hernando, A., Marín, P., Antonenko, A., Larin, V. and Torkunov, A. (1996). Patent: Método de codificación y marcado magnético de objetos, P9601993.

Vázquez, M. and Zhukov, A.P. (1996). Magnetic properties of glass-coated amorphous and nanocrystalline microwires. Journal of Magnetism and Magnetic Materials, 160, $223-228$.

Vázquez, M., Knobel, M., Sánchez, M.L., Valenzuela, R., Zhukov, A.P. (1997). Giant magneto-impedance effect in soft magnetic wires for sensor applications. Sensors and Actuators A, 59, 20-29.

Velazquez, J., Vázquez, M., Chen, D.X. and Hernando, A. (1995). Giant magneto-impedance in non magnetostrictive amorphous wires. Physical Review B, 50, 16737-16740.

Velázquez, J., Vázquez, M. and Zhukov, A.P. (1996). Magnetoelastic anisotropy distribution in glass-coated microwires. Journal of Materials Research, 11(10), 2499-2505.

Yoshizawa, Y., Oguma, S. and Yamanchi, K. (1988). New Fe-based soft magnetic alloys composed of ultrafine grain structure. Journal of Applied Physics, 64, 6044-6046. 